C. Wohlin, M. Xie and M. Ahlgren, "Reducing Time to Market through Optimization with Respect to Soft Factors", Proceedings IEEE Annual International Engineering Management Conference, pp. 116-121, Singapore, 1995. 


\section{Reducing Time to Market through Optimization with Respect to Soft Factors}

\author{
Claes Wohlin \\ Dept. of Communications \\ Systems \\ Lund University \\ Box 118 \\ S - 22100 Lund \\ Sweden
}

\author{
Min Xie \\ Dept. of Industrial and \\ Systems Engineering \\ National University of Singapore \\ Kent Ridge \\ Singapore 0511 \\ Republic of Singapore
}

\author{
Magnus Ahlgren \\ Q-Labs AB \\ IDEON Research Park \\ S-223 70 Lund \\ Sweden
}

\begin{abstract}
One of the most important measures of the productivity of an organization is the time to market of the products. There is a constant need to reduce it to meet the increasing demand of the customers and to gain more competitiveness. There are many technical issues that affect the time to market and sometimes they cannot be changed due to high cost of purchasing new equipment and the training of the employees. In a previous study, some soft factors and their impact on time to market in software development have been studied and an interesting conclusion is that they strongly affect the time to market and they are helpful in planning and controlling time to market. The focus in this paper is to study the implementation of the proposed model in reducing time to market through optimization of soft factors. The results are useful for management in their decision-making for controlling the time to market.
\end{abstract}

\section{Introduction}

Time to market is often depicted as one of the most important attributes to stay on the competitive edge. It is crucial for companies to find the market-window and beat its competitors to the market. An empirical study of delays in software projects is presented in [4] and a description of some critical factors for successful project implementation in general can be found in [3].

The ability of the software industry to keep its time schedules is often limited and cost overruns and late delivery are all too common. To improve this situation, new methods are needed to allow for better planning and control of software projects.

The focus in the software industry is often on new development methods and CASE tools, but the main reasons for the problems are often non-technical issues, see for example [1]. The objective here is to highlight the importance of some of the non-technical factors, which hereafter is referred to as soft factor. A soft factors is any factor which is hard to quantify by objective means, for example human factors as competence.

The soft factors have been included in a method for planning and controlling software projects, [5]. The method can be used for two main purposes:

- optimization of the soft factors based on requirements on effort and time to market,

- determination of effort and time to market when the soft factors are known in the forthcoming project.

The main objective with this paper is to highlight and exemplify how the soft factors can be used in the software industry to improve the prediction and delivery precision of software projects. In order to do this, the method is summarized briefly and a more detailed description can be found in [5].

The method is general and it is exemplified with a set of data collected from 12 large software projects. The data are presented in Section 2. An analysis of the data is discussed in Section 3, while a soft factor goodness value is discussed in Section 4. The goodness value forms the basis for the method including soft factors in the controlling and planning process. The use of the goodness value is discussed for optimization purposes in Section 5 and an illustration of the optimization procedure is presented in Section 6. The determination of effort and time to market based on the soft factors is briefly described in Section 7. Finally, some conclusions are presented in Section 8.

\section{Data collection}

The 12 projects are plotted in a diagram according to their time to market and $\log$ (effort), Figure 1. It is known, from earlier studies, that the relationship 
between time to market and effort is non-linear [1, 2], thus explaining the use of $\log$ (effort). It can be seen that the data points are scattered all over the diagram, which indicates that simple relationships between effort and time to market will be insufficient to explain the relationship.

A fast project is characterized by a low value of time to market / $\log$ (effort), i.e. time to market is normalized by manpower. This entity is denoted normalized time to market. In Figure 1 this implies that project 9 is the fastest project. This is based on the assumption that all hours put into a project are productive, and hence the ability of producing a lot of work in the shortest possible time is the basis for determining what a fast project is.

10 soft factors have been graded in the 12 projects. The factors are:

I. Competence

II. Product complexity

III. Requirement stability

IV. Staff turnover

V. Geographical distribution

VI. Methods and tools

VII. Time pressure

VIII.Information flow

IX. Priority

X. Project management

Each factor has been given a grade in the range 1-5. The hypothesis is that a high grade results in a fast project. The factors are discussed in more detail in [5]. The data are presented in Table 1, where the 10 factors are indicated as well although not formally defined. Some grades could not be determined based on the available information in terms of project reports and available personnel to interview.

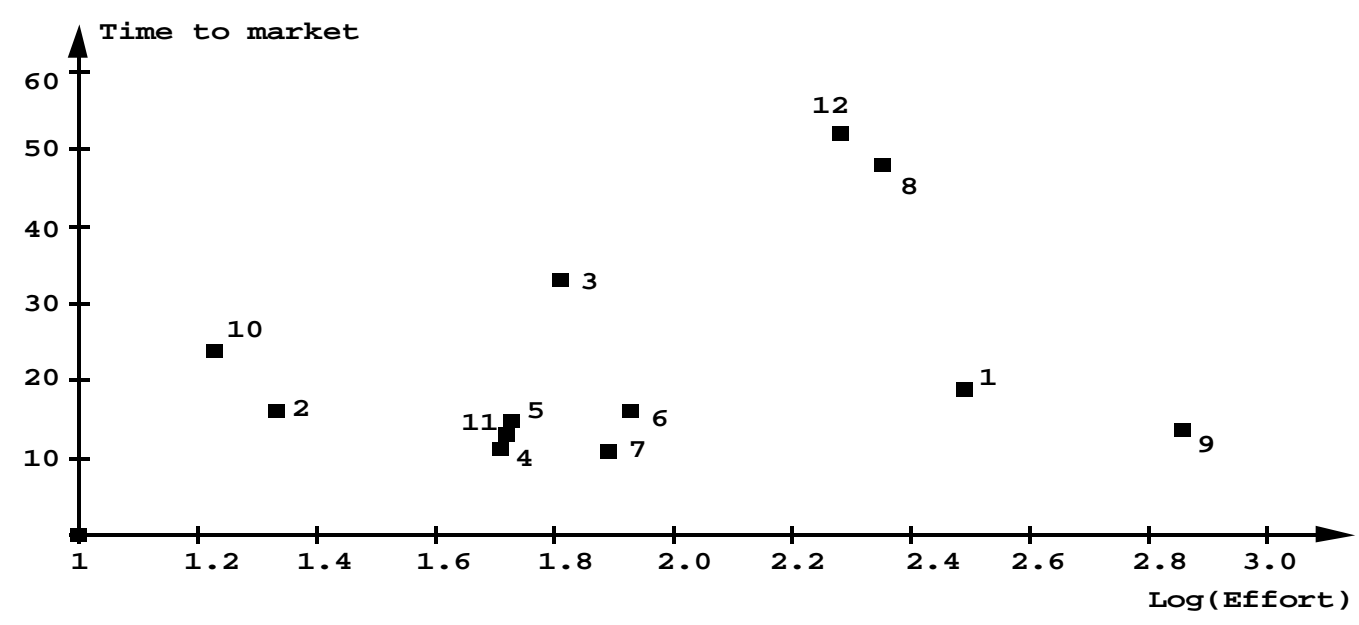

Figure 1. The relationship between log(effort) and time to market. method in the future.

\section{Data analysis} three groups are:

- Expected correlation: $\mathrm{C}$ ð - 0.4.

- No correlation: $-0.4<\mathrm{C}<0.4$

- Unexpected correlation: C Š 0.4 in the groups. The soft factors are placed as follows:

- Expected correlation:

Factor: III, VII, VIII and IX

- No correlation:

Factor: II, IV, VI and X

- Unexpected correlation:

Factor: I and V the soft factors.

The soft factors are, of course, correlated and an analysis has been made and the results are presented in [5]. At this stage of the method usage, it is judged that the correlation between some of the soft factors is not a critical factor and it is left for further refinement of the

The expectation based on the soft factors and the normalized time to market is that there should be a positive correlation between the normalized time to market and the grades for a particular soft factor. Therefore, a correlation study is conducted and the soft factors are divided into three groups based on the correlation. The objective was to divide the correlation into three equally large groups, but as it is doubtful if 0.33 can be regarded as a correlation it was decided to use 0.4 instead. The

Based on this division, the soft factors can be placed

This division forms the basis for deriving a soft factor goodness value for each software project, see section 3 , which then can be used for managing the influence of

Due to the scattering of the data points, as can be seen in Figure 1, classification of the projects is needed. 
Table 1. Project data for the 12 projects included in the study

\begin{tabular}{|l||c|c|c|c|c|c|c|c|c|c|c|c|}
\hline Soft factor/ Project & $\mathbf{1}$ & $\mathbf{2}$ & $\mathbf{3}$ & $\mathbf{4}$ & $\mathbf{5}$ & $\mathbf{6}$ & $\mathbf{7}$ & $\mathbf{8}$ & $\mathbf{9}$ & $\mathbf{1 0}$ & $\mathbf{1 1}$ & $\mathbf{1 2}$ \\
\hline \hline I. Competence & 3.5 & 3 & 4 & 3 & 4 & 2 & 3 & 4 & 2 & 3 & 2.5 & 3 \\
\hline II. Product complexity & 2 & 2 & 1 & - & - & - & 2 & 5 & 3 & 4 & 3 & 1 \\
\hline III. Requirem't stability & 3 & 4 & 2 & 3 & 2 & 4 & 4.5 & 1 & 3 & 1 & 2.5 & 2 \\
\hline IV. Staff turnover & 3 & 3 & 4 & 3 & 4 & 3 & 5 & 2 & 2 & 3 & 3 & 2 \\
\hline V. Geographical dist. & 1 & 2 & 1 & 2 & 2 & 1 & 1.5 & 5 & 1 & 3 & 2 & 1 \\
\hline VI. Methods and tools & 2.5 & 2.5 & 2 & 3 & 3 & 3 & 2 & 2 & 2 & 3 & 3 & 3 \\
\hline VI. Time pressure & 4.5 & 5 & - & 3 & 4 & 3 & 3 & 3 & 5 & 2 & 3.5 & 3 \\
\hline VIII. Information flow & 4.5 & 4 & 3 & - & - & 3 & 4 & 3 & 4 & 2 & 4.5 & 3 \\
\hline IX. Priority & 4 & 1 & 3 & 3 & 3 & 3 & 4 & 3 & 5 & 2 & 3.5 & 3 \\
\hline X. Project managem't & 4.5 & 3 & 3 & 2 & 3 & 2 & 4 & 4 & 5 & 2 & 4 & 4 \\
\hline
\end{tabular}

It is thought that there is no single relationship which explains the scattering of the data points. Therefore, the objective is to identify suitable classes and, for a particular project, place it in the correct class based on the soft factors. The number of data points is limited, hence it is not possible to divide them into a large number of classes, therefore a compromise must be accepted.

The projects can be divided into three classes: fast, normal and slow. The 12 projects were divided into classes based on an ocular inspection of Figure 1. Slow projects were: $3,8,10$ and 12 , normal projects: $1,2,5,6$ and 11 and finally fast projects: 4, 7 and 9. The classes and the lines describing them are illustrated in Figure 2. The objective is to try to relate new projects to these classes thus allowing better predictions to be made than by comparing with a mean value of all projects. The hypothesis is that the soft factors can be used to determine the class of the project.

A method to map the soft factors through a goodness value on to the three classes discussed above are presented below.

\section{A goodness value}

The first step is to assign significance values to the soft factors based on their correlation and the expectation. Significance values are assigned as follows:

- Expected correlation: 3

- No correlation: 2

- Unexpected correlation: 1

The motivation for choosing these significance values is given in [5]. To be able to predict time to market as well as to plan and control projects with respect to soft factors, the effect of the soft factors must be quantified. This implies that it must be possible to calculate a value which indicates the type of project to be obtained. A soft factor goodness value ( $G$ value) must be calculated. This is done in the following way:

- The grade of the soft factor (1-5, according to the assigned numbers) is multiplied by its significance value $(3,2$ or 1 , according to the grouping of the soft factors).

- The values from 1 above are summed for all the soft factors being graded.

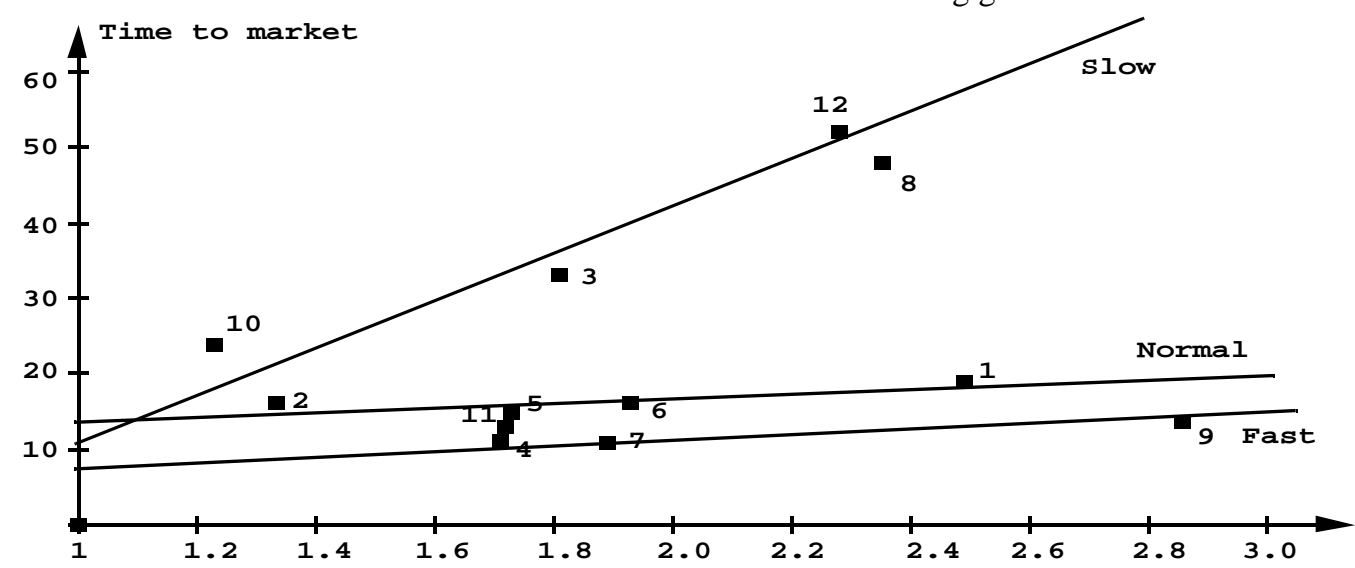

Figure 2. Classification of the projects into fast, normal and slow. 
- The sum from above is divided by the number of factors being graded (in our case a maximum of 10 factors). The $\mathrm{G}$ value is thus obtained.

The hypothesis is that the $G$ value indicates how good the soft factors are in a specific project. The $\mathrm{G}$ values for the 12 projects are shown in Table 2. It is comforting to see that the high $G$ values are mainly in the projects which were considered fast and vice versa. To enable a better analysis, the $G$ values must be divided into three groups as well to obtain a correspondence with the classification into fast, normal and slow projects.

In [5], it is described how the possible range of the $G$ values can be divided into three equally likely intervals. The intervals become:

- Fast projects: 7.0 ð G value ð 11.0

- Normal projects: $6.2<\mathrm{G}$ value $<7.0$

- Slow projects: 2.2 ð G value ð 6.2

The limits indicate that the projects should be grouped as follows:

- $\quad$ Fast projects: 1 and 7

- Normal projects: 2, 5, 6, 9 and 11

- Slow projects: $3,4,8,10,12$

This division, based on the soft factors through the $\mathrm{G}$ value, can be compared with the division above. It can be seen that most projects are placed in the same class of projects, although some of them are placed erroneous. The following can be noted as differences:

- Project 4 and 9 ought to be fast projects. The $\mathrm{G}$ value for project 9 is very close to the limits for the $G$ value and the erroneous placing is not viewed as critical. The wrong placing of project 4 is more critical as it is placed among the slow projects although it ought to be a fast project.

- Project 1 ought to be a normal project, although it is depicted as a fast project through the G-value. The value is, however, close to the limit and thus the placing is not that critical.

In total three projects are placed in the wrong class when comparing the actual placing of the projects, see Figure 1 and 2, and when basing the placing on the $G$ value. This result is quite satisfactory taken into account the normal uncertainty when determining schedules for software projects.

It must also be noted that correlation between the $\mathrm{G}$ values and the normalized time to market is -0.76 (or 0.72 using rank correlation). This correlation must be considered to be quite good.
The opportunity to optimize the soft factors to increase the probability to obtain a specific time to market is discussed in the next section, while the determination of the effort and time to market based on a number of predetermined soft factors are discussed in Section 5.

\section{Optimizing soft factors}

The basis for using the soft factors for planning and controlling purposes has been established. Thus, it is now possible to based on requirements on cost, primarily effort in terms of person-hours in software projects, and time to market to start using the knowledge between the soft factors and the actual outcome of time to market.

Another important issue is, of course, that the understanding of the soft factors has increased. The study and the method in particular result in knowledge which can be used to explain why it is impossible to conduct certain projects under some given conditions in the time frame required.

The optimization possibility does not only mean that it is possible to obtain a fast project, but to optimize in relation to the given circumstances. For example, if the time to market is non-critical then it is possible to use the project for learning purposes, and since three different lines are available to predict time to market it is possible to predict the time to market even in this type of project.

A more common situation is, however, that certain requirements are set on time to market and cost. The three lines can now be used to evaluate if the combination of effort and time to market is at all feasible, and if it is feasible then it is possible to discuss different combinations of the soft factors. The combination must give a $\mathrm{G}$ value which result in the "correct" line in relation to the requirements set. The procedure for soft factors optimization can be summarized in the following steps:

1. The cost (effort) or the time to market is determined based on requirements.

2. If only the cost or the time to market is determined, then some opportunities for the other parameter can be found from the lines in Figure 2.

3. It must be determined which line to aim for and hence the required range for the $G$ value is known through the division into the three intervals.

4. The determination of a required $G$ value results in an equation with 10 unknown soft factors, which must be combined in a way which results in the required $\mathrm{G}$ value.

Table 2. G values for the projects studied.

\begin{tabular}{|c||c|c|c|c|c|c|c|c|c|c|c|c|}
\hline Project & $\mathbf{1}$ & $\mathbf{2}$ & $\mathbf{3}$ & $\mathbf{4}$ & $\mathbf{5}$ & $\mathbf{6}$ & $\mathbf{7}$ & $\mathbf{8}$ & $\mathbf{9}$ & $\mathbf{1 0}$ & $\mathbf{1 1}$ & $\mathbf{1 2}$ \\
\hline \hline G value & 7.15 & 6.55 & 5.67 & 6.00 & 6.62 & 6.44 & 7.50 & 5.80 & 6.90 & 4.90 & 6.85 & 5.50 \\
\hline
\end{tabular}


5. If some of the 10 factors are predetermined due to the circumstances of the project, then the problem is reduced as the number of unknown factors is reduced.

6. The 10 factors are best determined in priority order, i.e. it is best to start with factors that contribute the most to the final $G$ value. Therefore, it is recommended to start with the soft factors having a significance value of three.

7. The soft factors having a significance value of two are studied next.

8. Finally, the soft factors having the lowest significance value are determined.

9. If the resulting $\mathrm{G}$ value indicates that the line fulfilling the requirement can be used, then it is just to go ahead and make sure that the soft factors are fulfilled in accordance with the expectation.

10. If the $\mathrm{G}$ value indicates a line which is unacceptable, it is necessary to either re-plan the project based on the line indicated or to influence the soft factors in such a way that another $G$ value which indicates the "correct" line is obtained.

This procedure can easily be followed, when trying to optimize the soft factors to achieve a certain time to market or effort.

\section{Soft factor optimization scenario}

The ten step procedure in the previous section is here followed to illustrate how it can be applied to optimize the soft factors in a particular project. If the procedure is followed, it is reasonable to believe that the probability for actually fulfilling the requirements set has increased than without taking the soft factors into account.

The following items illustrate how the soft factors can be optimized for a specific project:

1. The customer requires the software product to be delivered after 12 time units, see Figure 2.

2. The opportunities concerning effort can be found by studying Figure 2.

3. Our company wants to minimize the cost, in particular the effort spent on the development. Therefore, we must use the line for fast projects in Figure 2.
4. The soft factors are denoted $F_{I}$ to $F_{X}$ and gives rise to an equation, where the soft factors should be given values so that the $\mathrm{G}$-value is greater or equal to 7.0 .

5. None of the soft factors is predetermined, hence the equation discussed in the previous item is still valid.

6. The focus is first set on the factors with significance value of three. The requirement stability belongs to this group and hence negotiations are carried out with the customer, where it is agreed that in order to deliver within 12 time units, the customer is requested to keep the changes to a minimum, hence giving a good grade $\left(\mathrm{F}_{\mathrm{III}}=4\right)$, see Table 3 , where the order of the soft factors in Table 3 is the same as in Table 1 . The time pressure will be high $\left(\mathrm{F}_{\mathrm{VII}}=4\right)$, but this particular customer does not have the highest priority from a line management perspective. Thus, the project will only have normal priority from the management $\left(F_{I X}=3\right)$. Finally, the last factor in this group can be considered, i.e. information flow. Unfortunately, the project has to rely on the normal information procedure of the company $\left(\mathrm{F}_{\mathrm{VIII}}\right.$ $=3$ ).

7. The factors having significance values of two are considered next. By exploring the factors in this group further, it is obvious that it is not possible to only use people who have been at the company for a long time and who are not expected to leave, hence the staff turnover is assumed to be normal $\left(\mathrm{F}_{\mathrm{IV}}=3\right)$. The methods and tools follow the company standard $\left(\mathrm{F}_{\mathrm{VI}}=3\right)$. The project is, however, assigned to a project leader who has a good record, very good reputation and he is also trusted by the personnel, hence leading to a high grade $\left(\mathrm{F}_{X}=5\right)$. The complexity is estimated to be normal compared with what the company is used to developing $\left(\mathrm{F}_{\mathrm{II}}=3\right)$.

8. Finally, the factors having a significance value of one are studied. The competence in the project group will be normal based on knowledge about the available personnel $\left(\mathrm{F}_{\mathrm{I}}=3\right)$. The geographical distribution is unfortunately expected to be bad, i.e. the development will be spread over four organizations with sub-project leaders at each site. This renders a low grade for the geographical distribution $\left(\mathrm{F}_{\mathrm{V}}=1\right)$.

9. The $G$ value is calculated by first multiplying the grades in item 6 by the significance value 3 , the grades in item 7 by the significance value 2 and finally the grades in item 8 by the significance value 1. The $G$ value is then derived by summing the results of the multiplications and then by dividing

Table 3. Soft factors and their grades.

\begin{tabular}{|l||c|c|c|c|c|c|c|c|c|c|}
\hline Soft factor & I & II & III & IV & V & VI & VII & VIII & IV & X \\
\hline \hline Grade & 3 & 3 & 4 & 3 & 1 & 3 & 4 & 3 & 3 & 5 \\
\hline
\end{tabular}


the sum with the number of grades, see Table 1 . The $\mathrm{G}$ value becomes 7.4, which means that the fast line can be used, hence fulfilling the requirements set in the best possible way.

10. This is not relevant in this particular example.

Based on the $\mathrm{G}$ value, it is concluded that the probability of success is high and that all possible precautions have been taken to ensure control over the soft factors. The actual outcome of the factors must be monitored and the experience from the project must be included in the experience base of the company as the project is finalised.

It should be noted that an average project, with grades 3 for all the soft factors will obtain a $G$ value of 6.6. This is in correspondence with the expectation, when comparing with the division of the $G$ value into three intervals.

\section{Project planning}

The soft factors can also easily be used for project planning purposes. When the circumstances of a particular project is known, then it should be an easy task to determine the soft factors. Moreover, it is easy to calculate the $\mathrm{G}$ value, which then could be used to find a possible combination between effort and time to market, see Figure 2.

This way of using the knowledge of the soft factors will improve the estimation of effort and time to market. This is shown in [5], where four projects are used to evaluate the proposed procedure. The estimations for all four projects are improved by incorporating the knowledge of the soft factors in the estimation procedure. Therefore, the understanding and the usage of the soft factors are crucial to improve project planning and control.

\section{Conclusions}

Time to market is an important process quality attribute and it is hence important both to improve it and to improve the predictability of it. The method presented is based on a study of 12 projects, but the ideas and the way to incorporate the soft factors in the planning and controlling of software projects are general.

The method has been presented in detail in [5] and the opportunities to apply it in practice has been highlighted here. It has been shown how the method can be used to control the soft factors to optimize a particular project with respect to time to market. It has also been emphasized how the understanding and knowledge of the soft factors can be used to enable better planning and control of software projects.
It should be noted that it is unlikely that full control of the soft factors can be achieved. In particular, it is not reasonable to expect that the method can be used to produce results with any desired statistical significance. The main reason is that only one data point is obtained for each project and the data are aging. The latter implies that due to changes, hopefully improvements, in the organizations the data become obsolete. Therefore, it is important to discard projects from use in the method as they no longer give a representative picture of how software projects are conducted.

The method can probably be improved by taking the correlation between the soft factors into account and also by learning more about the soft factors through an enlarged study. The latter implies that the method must be further investigated as it is applied in new projects.

Finally, it can be concluded that methods are needed which take non-technical issues into account. The problems with missed deadlines and budgets in the software industry can not solely be explained with technical issues and therefore a better understanding and control of the non-technical issues are essential to be on the competitive edge. A similar method can probably be applied in other engineering disciplines experiencing the same type of problems as the software industry.

\section{Acknowledgement}

We would like to thank the organizations and the people who provided us with the data.

\section{References}

[1] B. Boehm, Software Engineering Economics, Prentice-Hall, Englewood Cliffs, N.J., USA, 1981.

[2] B. Kitchenham, "Management Metrics", in Software Reliability - Achievement and Assessment, Littlewood B. (ed), Blackwell Scientific Publication, 1987.

[3] J. K. Pinto and D. P. Slevin, "Critical Factors in Successful Project Implementation", IEEE Transactions on Engineering Management, Vol. 34, No. 1, pp. 2227, 1987.

[4] M. van Genuchten, "Why is Software Late? An Empirical Study of Reasons for Delay in Software Development", IEEE Transactions on Software Engineering, Vol. 17, No. 6, pp. 582-590, 1991.

[5] C. Wohlin and M. Ahlgren, "Soft Factors and Their Impact on Time to Market", Accepted for publication, to appear in Software Quality Journal, 1995. 